\title{
Comparison of effects of classic LMA, cobraPLA and V-gel rabbit on QTc interval
}

\author{
Toman $\mathrm{H}^{1}$, Erbas $\mathrm{M}^{1}$, Kiraz $\mathrm{HA}^{1}$, Sahin $\mathrm{H}^{1}$, Ovali MA², Uzun $\mathrm{M}^{2}$ \\ Department of Anesthesiology and Reanimation, Canakkale Onsekiz Mart University, Faculty of Medicine, \\ Canakkale, Turkey. huseyintoman01@hotmail.com
}

\begin{abstract}
AIM: We aimed to compare the effects of three different supraglottic airway devices, the classic LMA, PLA, and V-gel, on hemodynamics and QTc in rabbits under general anesthesia.

METHOD: The rabbits were divided into four groups: Group $C(n=5)$ control group with no airway device used, Group L ( $n=5$, classic LMA), Group P ( $n=5$, CobraPLA) and Group V ( $n=5$, V-gel-rabbit). Basal values of heart rate (HR), mean arterial pressure (MAP) and ECG for QTc interval were measured and the measurements were evaluated at 1, 5, 15, and 30 minutes after inserting the airway device

RESULTS: The values of HR, MAP and QTc in Group $V$ at minutes 1 and 5 were significantly different to those in Group $L$ and Group P $(p<0.05)$.

DISCUSSION: The classic-LMA and cobraPLA cover a wide part of the perilaryngeal area with cuffs inflated to about $60 \mathrm{cmH}_{2} \mathrm{O}$ of pressure resulting in mucosal compression. As V-gel rabbit does not have a cuff, it covers a smaller part of the laryngopharyngeal area, and thus does not cause mucosal compression, and the hemodynamic response is weaker.

CONCLUSION: When comparing hemodynamic responses to other supraglottic airway devices, the response to V-gel rabbit is minimal and we consider that similar studies using the I-gel on humans are required (Fig. 5, Ref. 31). Text in PDF www.elis.sk. KEY WORDS: LMA, cobraPLA, V-gel, supraglottic airway, QTc.
\end{abstract}

\section{Introduction}

Supraglottic airway devices are frequently used in general anesthesia applications, especially for outpatient and short surgeries. The use of supraglottic airway devices reduces the need for muscle relaxants, shortens the duration to discharge, and allows the patients to return early to their social and occupational activities $(1,2)$.

Various types of supraglottic airway devices are used in relation to age group, intubation difficulty, and elective and emergency situations in a reliable fashion $(1,3,4)$. Airway devices may have inflatable cuffs similar to those in cobraPLA and LMA and may be produced from thermoplastic elastomer appropriate to the anatomy of the perilaryngeal area, similar to I-gel, while non-inflatable supraglottic airway devices are also available $(5,6)$.

No matter how much endotracheal intubation has gained acceptance as the gold standard for reliable airways in general an-

${ }^{1}$ Department of Anesthesiology and Reanimation, Canakkale Onsekiz Mart University, Faculty of Medicine, Canakkale, Turkey, and ${ }^{2}$ Department of Physiology, Canakkale Onsekiz Mart University, Faculty of Medicine, Canakkale, Turkey

Address for correspondence: $\mathrm{H}$. Toman, MD, Department of Anesthesiology and Reanimation, Canakkale Onsekiz Mart University, Faculty of Medicine, 17100, Canakkale, Turkey.

Fax: +90.286.2635956, Phone: +90.505 .4505956$

Acknowledgement: This study was supported by Çanakkale Onsekiz Mart University Scientific Research Project Found with TSA-2013-80 project number. esthesia $(1,7)$, laryngoscopy and endotracheal intubation cause an unwanted reflex of sympathetic stimulation and increase the plasma catecholamine levels $(1,8,9)$. The increase in plasma catecholamine levels may cause unwanted hemodynamic responses such as hypertension, tachycardia, myocardial ischemia, reduction in myocardial contractility, and ventricular arrhythmia (1, 9). Ventricular arrhythmia may lengthen the QT interval (9-11), while arrhythmia occurring with lengthened QT interval may even cause sudden cardiac death (9-11).

As supraglottic airway devices do not require laryngoscopy and provide reliable airway without tracheal intubation, they induce a weaker hemodynamic response which is beneficial for patients with cardiovascular disease (12).

This study was aimed at comparing three different supraglottic airway devices, classic LMA, cobraPLA, and V-gel rabbit (13) produced by I-gel to be appropriate for rabbit anatomy, in terms of their effects on MAP, HR, and QTc interval.

\section{Material and method}

This study used 20 adult New Zealand white rabbits weighing 2.5-3.5 kg. Experiments received required permissions from Canakkale Onsekiz Mart University Animal Experiments Ethics Committee and were completed at Canakkale Onsekiz Mart University Experimental Research Center. Before the study had begun, the rabbits underwent clinical examinations for behavior, and respiratory and cardiovascular systems, while no negatives 
had been found in the animals included in the study.

All experiments took place between 09:00 and 16:00. During the experiments, animals were fed standard feed, and had constant access to water. The temperature of the housing was $21 \pm 2$ ${ }^{\circ} \mathrm{C}$ and the light was regulated to $12 / 12$-hour light/dark intervals.

Twenty rabbits were randomly divided into four groups:

Group C $(n=5)$ : Control group without airway device. Respiration was supported by a face mask.

GroupL( $n=5)$ : Groupused classic LMAairway device(Size 1, La Premiere Plus, Amstrong Medical Ltd. Coleraine, Northern Ireland)

Group $P(n=5)$ : Group used cobraPLA airway device (Size $1 / 2$, Pulmodyne Inc., Indiana, USA)

Group V $(n=5)$ : Group used V-gel rabbit airway device (R-3, Docsinnovent ${ }^{\mathbb{R}}$ Ltd. London, UK)

The front and rear extremities of the rabbits to be held in crocodile clips to obtain electrocardiographic recording (ECG) were shaved. Before the procedure the rabbits were starved for 12 hours. To prevent loss of heat during anesthesia a blanket set to $39^{\circ} \mathrm{C}$ was spread on the ground. The rabbits' initial ECG (Digital ECG system Poly-Spectrum-8/E, Neurosoft Ltd. 5, Voronin str., Ivanovo, Russia) was recorded. Rectal temperature was taken. A 24-gauge catheter (Bicakcilar Tibbi Cihazlar AŞ, Istanbul, Turkey) was used to enter the left main auricular artery, and invasive blood pressure was monitored (PETAŞ® KMA 800, Ankara, Turkey). Thirty minutes of general anesthesia was planned for the rabbits and $5 \mathrm{mg} / \mathrm{kg}$ xylazine (Rompun ${ }^{\circledR}$, Bayer Healthcare LLC) and 30 $\mathrm{mg} / \mathrm{kg}$ ketamine HCL (Ketasol, Richter Pharma AG) was administered in the quadriceps femoris. Pedal and palpepral reflexes were checked and a 26-gauge catheter (Bicakcilar Tibbi Cihazlar AŞ, Istanbul, Turkey) was inserted in the right ear's lateral vein. The rabbits were supported with $50 \% \mathrm{O}_{2}$ and $50 \%$ air mix through a face mask and Mapleson $\mathrm{C}$ pediatric ventilation cycle (Morton Medikal San Tic Ltd, Izmir, Turkey). When spontaneous respiration became shallow, all rabbits had an airway device inserted by the same anesthetist. For the purpose of facilitating the procedure of inserting the airway device, another anesthetist pulled the rabbit's tongue to the lateral side of its mouth (Fig. 1).

\section{ECG recordings}

Electrocardiogram recordings were completed according to the method reported by Uzun et al (14). Through electrodes on

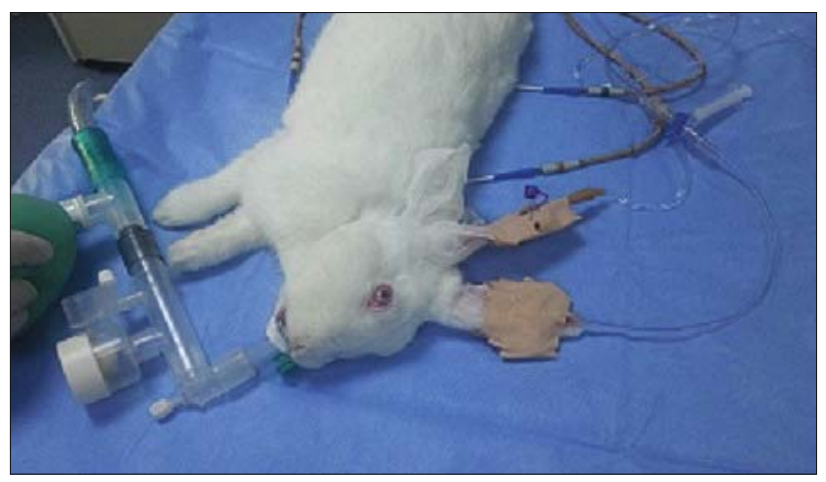

Fig. 1. The V-gel rabbit placed in a New Zealand white rabbit.

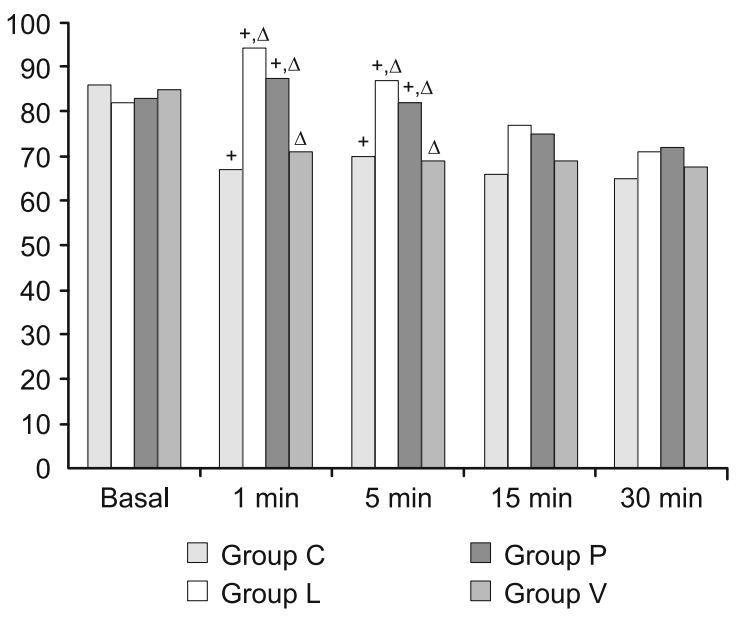

Fig. 2. Mean arterial pressure (MAP) values $(\mathrm{mmHg})$ in groups. $\uparrow, \Delta$ : It was observed that at minutes 1 and 5, MAP increased in Group L and Group P compared to group $C$ and Group V $(p<0.05)$.

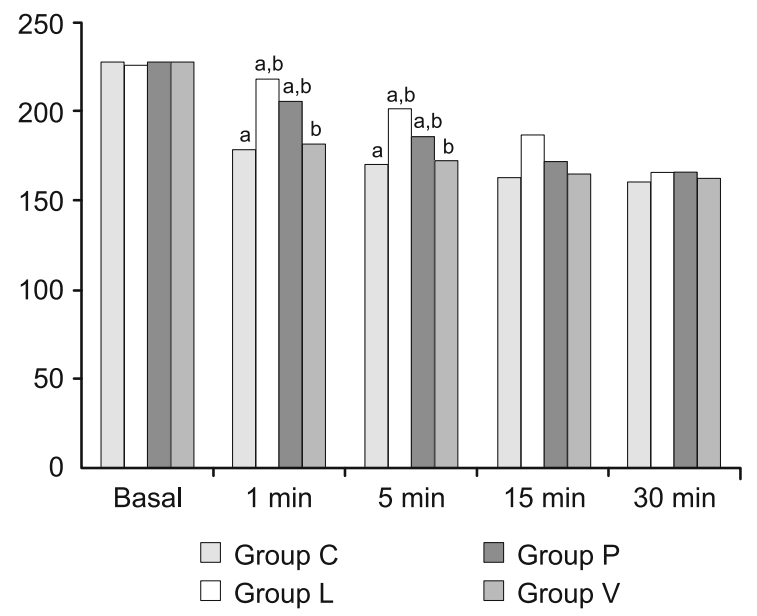

Fig. 3. QTc interval values (msec) in groups. a, b: It was observed that at minutes 1 and 5, QTc intervals increased in Group $L$ and Group P compared to Group C and Group V $(\mathbf{p}<\mathbf{0 . 0 5})$.

the extremities, measurements were recorded for basal values and repeated at 1, 5, 15 and 30 minutes after the insertion of airway device. ECG recordings were saved in a digital form with $1 \mathrm{mV}$ $=20 \mathrm{~mm}$, speed $50 \mathrm{~mm} / \mathrm{sn}$ and filter $(35 \mathrm{~Hz})$ used to record DI, DII, DIII, aVR, aVL, and aVF derivations. The QT interval was calculated as time from the start of $\mathrm{Q}$ wave to the end of $\mathrm{T}$ wave. Corrected QT interval (QTc) was calculated according to a formula derived by Bazett $(14,15)$.

\section{Maintenance of anesthesia}

All experimental animals were linked to an anesthesia machine (Anesthesia Machine w/O $\mathrm{O}_{2}$ Flush Model M3000PK Parkland Scientific Lab and Research Device. Florida, USA) and breathed with manual assistance. Anesthesia was maintained by providing $50 \%$ oxygen and $50 \%$ air mix with 1 MAC (Minimum alveolar concentration) isoflurane. The rabbits were manually ventilated to a respiration rate of about $40 / \mathrm{min}$, appropriate for rabbit physi- 


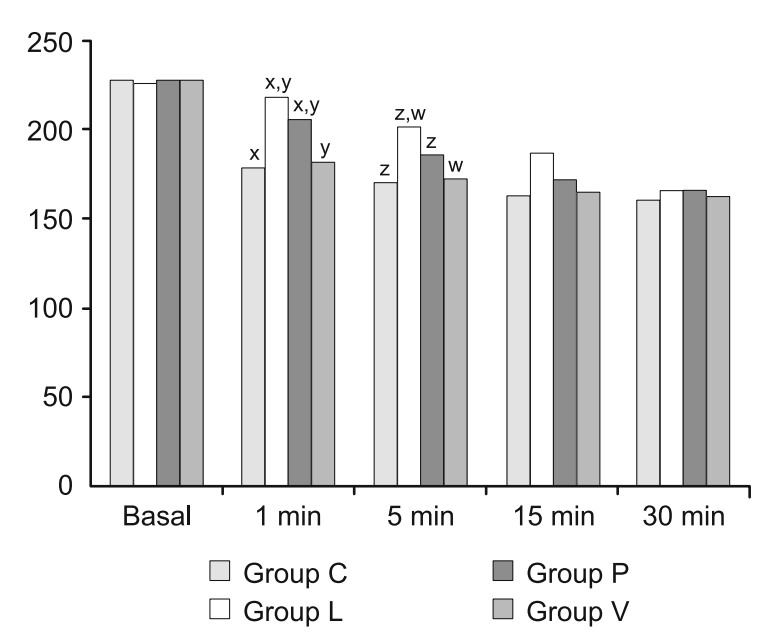

Fig. 4. Heart rate (HR) values (bpm) in groups. $x, y$ : It was observed that at minute $1, H R$ increased in Group $L$ and Group $P$ compared to Group $\mathrm{C}$ and Group V $(\mathrm{p}<\mathbf{0 . 0 5})$, z: It was observed that at minute 5, HR increased in Group $L$ and Group P compared to Group $\mathrm{C}(\mathrm{p}<$ $0.05)$, w: It was observed that at minute 5,HR increased in Group $L$ compared to Group V $(p<0.05)$.

ology, and pressure of $15 \mathrm{cmH}_{2} \mathrm{O}$ (about $10 \mathrm{ml} / \mathrm{kg}$ ) by the same anesthetist. MAP, HR, and I, II, III, aVR, aVL and aVF derivations of ECG were recorded at basal values and at 1, 5, 15 and 30 minutes after supraglottic airway device had been inserted. At the end of 30 minutes, the rabbits with sufficient respiration effort were extubated, while those without sufficient respiration were assisted for a little longer.

\section{Statistical analysis}

The data obtained were evaluated using SPSS 16 statistical program (SPSS Inc. Chicago USA) and significance was accepted at $\mathrm{p}<0.05$. To compare the groups in terms of the initial HR, MAP, blood gas values and QTc interval, at 1, 5, 15 and 30 minutes after insertion of the airway device, one-way ANOVA (Tukey's T-test) was used.

\section{Results}

The average weight of the rabbits included in the study was similar. During the study at minutes 1 and 5 after the insertion of supraglottic airway device in the 4 groups of rabbits (none inserted in the control group), the MAP values in the control group and V-gel group were similar. At same intervals, the difference between the control and V-gel rabbit groups and cobraPLA and classic LMA groups in terms of MAP was found to be similar ( $p$ $<0.05$ ). Additionally at minutes 1 and 5 while the MAP values of the classic LMA and cobraPLA groups were increased compared to basal values, there was no significant difference between the two groups. At minutes 15 and 30 after the insertion of supraglottic device there was no significant difference seen in MAP values compared to basal values (Fig. 2).

The basal QTc intervals and those at 1, 5, 15 and 30 minutes after intubation in the 4 groups were compared. There was no sig- nificant difference between the basal QTc values in all groups. At minutes 1 and 5 after the insertion of supraglottic airway device, the QTc values of the control group and V-gel rabbit group were seen to be similar. At same intervals, there was a significant difference between the control and V-gel groups and cobraPLA and classic LMA groups in terms of QTc interval $(\mathrm{p}<0.05)$. Additionally while the QTc intervals at minutes 1 and 5 in the classic LMA and cobraPLA groups were increased compared to basal values, there was no significant difference between the two groups. In all groups at minutes 15 and 30 after the insertion of supraglottic airway device, there was no significant difference between QTc values (Fig. 3).

The basal HR values and those at 1, 5, 15 and 30 minutes after intubation in the 4 groups were compared. In the rabbits in the four groups there was no significant difference observed between basal HR values. At minute 1 after the insertion of supraglottic airway devices, HR in the control and V-gel groups were similar. Again there was a significant difference observed between control and V-gel groups and cobraPLA and classic LMA groups at minute $1(\mathrm{p}<0.05)$.

At minute 5, there was a significant difference between the classic LMA group and control and V-gel rabbit groups $(\mathrm{p}<0.05)$. Again at minute 5 while the difference between the control group and cobraPLA group was significant in terms of HR $(p<0.05)$, there was no significant difference observed between the cobraPLA group and V-gel rabbit group. Additionally at minute 5, the HR values for the classic LMA and cobraPLA groups were similar. In all groups there was no significant difference in terms of HR 15 and 30 minutes after the insertion of supraglottic airway device (Fig. 4).

\section{Discussion}

In this study we observed that out of supraglottic airway devices, the classic LMA and cobraPLA increased the MAP and $\mathrm{HR}$, and lengthened the QTc interval compared to V-gel rabbit. We determined that the V-gel affected the MAP, HR, and QTc intervals less than cobraPLA and classic LMA, almost to the level of the control group.

Supraglottic airway devices have been used for nearly 30 years in surgical interventions in outpatients, patients requiring shortterm general anesthesia or in those requiring emergency airway $(16,17)$. Within this time, many different supraglottic airway de-

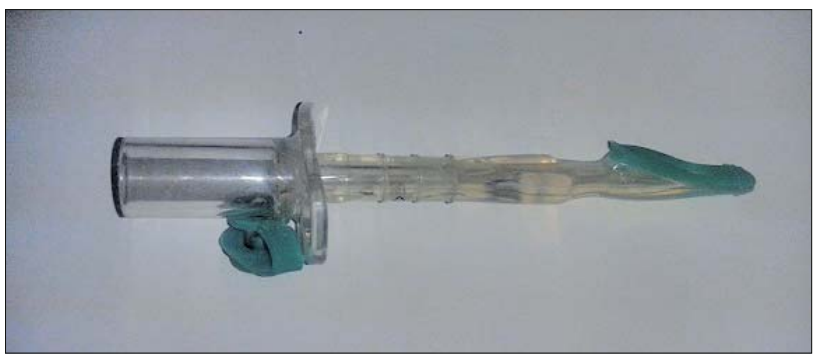

Fig. 5. V-gel rabbit. 
vices have been developed (17). The classic LMA was the first supraglottic airway device used (18). The classic LMA is a short silicon rubber tube similar to an endotracheal tube and linked to its tip is a flat mask with an ellipsoidal inflatable cuff (19). During the insertion, the epiglottis enters the tube, and in order to prevent airway obstruction the classic LMA has two vertical rubber grills (19). CobraPLA has a balloon structure and has completely different properties compared to the classic LMA in terms of the place of insertion (20). The inflated cuff is placed in the perilaryngeal region a little above the airway; the distal grated cobra head sits fully on the soft tissue and epiglottis and opens the airway (21). Igel is made of high-quality medical thermoplastic elastomer similar to soft gel and is not inflatable (1). When inserted, the soft gellike part takes a shape appropriate to the human pharynx, larynx, and perilaryngeal region $(1,17)$. V-gel rabbit, similar to I-gel, is produced from medical thermoplastic elastomer appropriate for rabbit anatomy (Fig. 5) (13).

At different stages of anesthetic applications, different hemodynamic responses may be seen. Especially during general anesthesia the lengthened QT interval may be seen as being linked to the increase in activity of autonomic nervous system and sympathoadrenergic reaction (22). The changes in the sympathoadrenergic activity, along with the development of hemodynamic response due to fear of surgery, agents used for anesthesia induction maintenance, intervention to provide reliable airway, the incision, and postoperative pain can enhance the rhythm disorders observed on electrocardiography $(23,24)$.

The hemodynamic response to the provision of reliable airway by means of supraglottic airway device in general anesthesia is minimal when compared to the response to laryngoscopy used for endotracheal intubation, insertion of the tube and inflation of the cuff within the trachea (25). While the hemodynamic response to the insertion of classic supraglottic LMA airway device is weaker than that due to intubation, there may be a partial hemodynamic response linked to catecholamine discharge (26).

When compared with endotracheal intubation, the supraglottic airway devices have advantages, such as reduced use of anesthetic agents, more stable hemodynamic response, reduced cough during revival, and less intense throat pain and irritation (1).

In their study on supraglottic airway devices, Dahaba et al determined that the hemodynamic response was proportional to cuff pressure and amount of air given. For example, while a size 5 laryngeal tube-suction cuff is inflated by $80 \mathrm{ml}$ air, again a size 5 Proseal-LMA is inflated with $40 \mathrm{ml}$ of air (4). Studies comparing cobraPLA and classic LMA have found that although the cobraPLA cuff is inflated by a nearly twice greater volume $(27,28)$, the increase in hemodynamics is minimal compared to classic LMA (27). Although not significantly different in our study, the HR, MAP and QTc values for classic LMA were higher than those for cobraPLA. In spite of high volume, similar hemodynamic effects may be due to the location of the cuff of the airway device, namely the oropharynx for cobraPLA and pharyngolaryngeal region for classic LMA.

To prevent air leaks in many supraglottic airway devices like classic LMA and cobraPLA, the cuff pressure should be around 60
$\mathrm{cmH}_{2} \mathrm{O}(29,30)$. In a study using silicon microchips on the supraglottic airway devices where they touch the oropharyngeal wall, Brimacombe et al showed that when mucosal perfusion pressure is higher than $34 \mathrm{cmH}_{2} \mathrm{O}$ there is a reduction in capillary blood flow $(30,31)$. Tissue damage occurs with a reduction in capillary blood flow (2). Here the tissue damage and throat pain appearing with hemodynamic response may be explained by the cuff pressure and capillary perfusion pressure in the pharyngeal mucosa.

In our study, at minutes 1 and 5 after the insertion of classic LMA and cobraPLA we observed a lengthened QTc and increased MAP and HR, while in the V-gel rabbit group no change was observed. This situation may be explained by the the facts that $\mathrm{V}$-gel rabbit fits the anatomy of the perilaryngeal region and it lacks the cuff.

This study is the first to compare the effects of supraglottic airway device on QTc interval. In our study, the classic LMA and cobraPLA caused greater sympathetic stimulation and the QTc intervals were significantly longer than in the other groups. This situation may be related to greater difficulty encountered when inserting the classic LMA and cobraPLA supraglottic devices due to their mismatch with rabbit anatomy. However we observed that the V-gel rabbit, produced to fit the rabbit anatomy, affected both the hemodynamic response and QTc interval to an extent that is too little to be important. Additionally the effect of cuff pressure of classic LMA and cobraPLA on part of the mucosa in the perilaryngeal region may cause an ischemic situation causing a greater release of catecholamine. In V-gel rabbit there is no cuff and thus we believe the minimal pressure exerted on oropharyngolaryngeal tissues may result in a weaker sympathetic response.

\section{Conclusion}

Although in general, the endotracheal intubation is considered to be the most reliable device for providing airway safety during anesthesia, we recommend to use supraglottic airway devices in patients with cardiovascular disease, namely in order to minimize the unwanted hemodynamic responses (lengthened QTc interval). Among these, the supraglottic airway devices, namely the non-cuff I-gel in humans and V-gel rabbit in rabbits may induce minimal stress response.

\section{References}

1. Das A, Majumdar S, Mukherjee A et al. I-gel in Ambulatory Surgery: A Comparison with LMA-ProSeal in Paralyzed Anaesthetized Patients. J Clin Diagn Res 2014; 8 (3): 80.

2. Aydogmus MT, Eksioglu B, Oba S, Ünsal O, Turk HSY, Sinikoglu SN, Tug A. Comparison of laryngeal mask airway supreme and laryngeal mask airway proseal for laryngopharyngeal trauma and postoperative morbidity in children. Rev Bras Anestesiol 2013; 63 (6): 445-449.

3. Kosucu M, Eroglu A, Besir A, Cansu A. Using Proseal LMA and I-gel for difficult airway management in patient with diffuse tracheal stenosis and pulmonary artery sling. Bratisl Lek Listy 2013; 114 (7): 418-420.

4. Dahaba AA, Prax N, Gaube W, Gries M, Rehak PH, Metzler H. Haemodynamic and catecholamine stress responses to the Laryngeal Tube Suc- 
tion Airway and the Proseal Laryngeal Mask Airway. Anaesthesia 2006; 61 (4): 330-334.

5. Uppal V, Fletcher G, Kinsella J. Comparison of the i-gel with the cuffed tracheal tube during pressure-controlled ventilation. $\mathrm{Br} \mathrm{J}$ Anaesth 2009; 102 (2): 264-268.

6. Janakiraman C, Chethan DB, Wilkes AR, Stacey MR, Goodwin N. A randomised crossover trial comparing the i-gel supraglottic airway and classic laryngeal mask airway. Anaesthesia 2009; 64 (6): 674-678.

7. Dreher M, Kluge S. Airway devices in the intensive care unit. Pneumologie 2014; 68 (6): 371-377.

8. Bullington J, Perry SM, Rigby $\mathbf{J}$ et al. The effect of advancing age on the sympathetic response to laryngoscopy and tracheal intubation. Anesth Analg 1989; 68 (5): 603-608.

9. Mizrak A, Kocamer B, Deniz H, Yendi F, Oner U. Cardiovascular changes after placement of a classic endotracheal tube, double-lumen tube, and Laryngeal Mask Airway. J Clin Anesth 2011; 23 (8): 616-620.

10. Rettenbacher MA, Eder-Ischia U, Bader A et al. QTc variability in schizophrenia patients treated with antipsychotics and healthy controls. J Clin Psychopharmacol 2005; 25: 206-210.

11. Basso C, Maron BJ, Corrado D, Thiene G. Clinical profile of congenital coronary artery anomalies with origin from the wrong aortic sinus leading to sudden death in young competitive athletes. J Am Coll Cardiol 2000; 35: 1493-1501.

12. Oczenski W, Krenn H, Dahaba AA et al. Hemodynamic and Catecholamine Stress Responses to Insertion of the Combitube [registered sign], Laryngeal Mask Airway or Tracheal Intubation. Anesth Analg 1999; 88: 1389-1394.

13. Crotaz IR. Initial feasibility investigation of the v-gel airway: an anatomically designed supraglottic airway device for use in companion animal veterinary anaesthesia Vet Anaesth Analg 2010; 37 (6): 579-580.

14. Uzun M, Karaca M, Topçu B, Kurt Y. The Effects of Intravenous Oxytocin and Methylergonovine Administration on QT Interval in Rabbits. Kafkas Uni Vet Fak Derg 2009; 15 (3): 351-356.

15. Bazett HC. An analysis of the time relations of electrocardiograms. Heart 1920; 7:353.

16. Sharma V, Verghese C, McKenna PJ. Prospective audit on the use of the LMA-Supreme for airway management of adult patients undergoing elective orthopaedic surgery in prone position. Br J Anaesth 2010; 105: 228-232.

17. Goyal R., Shukla RN, Kumar G. Comparison of size 2 i-gel supraglottic airway with LMA-ProSeal and LMA-Classic in spontaneously breathing children undergoing elective surgery. Paediatr Anaesth 2012; 22 (4): 355-359.
18. Agro FE, Cataldo R, Mattei A. New devices and techniques for airway management. Minerva Anestesiol 2009; 75 (3): 141-149.

19. Jones JR. Laryngeal mask airway: an alternative for the difficult airway. AANA J 1995; 63 (5): 444-449.

20. Hung O, Law JA. Advances in airway management. Can J Anesth 2006; 53: 628-631.

21. Szmuk P, Ghelber O, Matuszczak M, Rabb MF, Ezri T, Sessler DI. A prospective, randomized comparison of cobra perilaryngeal airway and laryngeal mask airway unique in pediatric patients. Anesth Analg 2008; 107 (5): 1523-1530.

22. Lindgren $\mathbf{L}$. ECG changes during halothane and enflurane anaesthesia for E. N. T surgery in children. Br J Anaesth 1996; 51: 219-224

23. Pernerstorfer T, Krafft P, Fitzgerald RD, Krenn CG, Chiari A, Wagner O, Weinstabl C. Stress response to tracheal intubation: direct laryngoscopy compared with blind oralintubation. Anaesthesia 1995; 50 (1): $17-22$.

24. Michaloudis D, Fraidakis O, Lefaki T, Dede I, Kanakoudes F, Askitopoulou H, Pollard BJ. Anaesthesia and the QT interval in humans the effects of isoflurane and halothane. Anaesthesia 1996; 51: 219-224.

25. Siddiqui NT, Khan FH. Haemodynamic response to tracheal intubation via intubating laryngeal mask airway versus direct laryngoscopic tracheal intubation. J Pak Med Assoc 2007; 57 (1): 11-14.

26. Wilson IG, Fell D, Robinson SL, Smith G. Cardiovascular responses to insertion of the laryngeal mask. Anaesthesia 1992; 47: 300-302.

27. Chung CJ, Lee KH, Choi SR, Kim DC, Lee SC. Comparison of the CobraPLA and the LMA Classic airway devices during volume-controlled ventilation in children. Korean J Anesthesiol 2008; 55 (2): 145-149.

28. Galvin EM, van Doorn M, Blazquez J, Ubben JF, Zijlstra FJ, Klein J, Verbrugge SJ. A randomized prospective study comparing the Cobra Perilaryngeal Airway and Laryngeal Mask Airway-Classic during controlled ventilation for gynecological laparoscopy. Anesth Analg 2007; 104 (1): 102-105.

29. Bergmann I, Crozier TA, Roessler $M$ et al. The effect of changing the sequence of cuff inflation and device fixation with the LMA-Supreme (R) on device position, ventilatory complications, and airway morbidity: a clinical and fiberscopic study. BMC Anesthesiol 2014; 14 (1): 2.

30. Brimacombe J, Keller C, Puehringer F. Pharyngeal mucosal pressure and perfusion. A fiberoptic evaluation of the posterior pharynx in anesthetized adult patients with a modified cuffed oropharyngeal airway. Anesthesiology 1999; 91: 1661-1665.

31. Brimacombe J, Keller C, Roth W, Loeckinger A. Large cuff volumes impede posterior pharyngeal mucosal perfusion with the laryngeal tube airway. Can J Anaesth 2002; 49: 1084-1087. 